\title{
Novel Optical Antenna Designs of Comb Shaped Split Ring Architecture for NIR and MIR Enhanced Field Localization
}

\author{
Veli Tayfun Kilic, ${ }^{1, *}$ Vakur B. Erturk, ${ }^{1}$ and Hilmi Volkan Demir ${ }^{1,2}$ \\ ${ }^{\prime}$ Department of Electrical and Electronics Engineering, Department of Physics, and UNAM-Institute of Materials Science and Nanotechnology, \\ Bilkent University, Ankara 06800, Turkey \\ ${ }^{2}$ School of Electrical and Electronic Engineering, School of Physical and Mathematical Sciences, Nanyang Technological University, Nanyang \\ Avenue, Singapore 639798, Singapore \\ vtkilic@ee.bilkent.edu.tr
}

\begin{abstract}
We demonstrated NIR/MIR resonance behavior in optical antennas of comb-shaped split-ring resonators enabling substantially larger field enhancements than single/array of dipoles with the same side length, despite their simple architecture.
\end{abstract}

Interaction between conduction electrons on metals with incident electromagnetic field results in collective electron oscillations on metal surfaces. These plasmonic interactions are of fundamental importance because they allow for confining fields beyond the diffraction limit [1]. As a result, today plamonics is exploited for various applications including optical transmission, spectroscopy and sensors.

Optical antennas make an important class of plasmonic structures. Different from the plasmonic structures of waveguides and transmission apertures, optical antennas generate hot spots at very high intensities in a region beyond the diffraction limit [2]. Therefore, optical antennas are useful for a number of important applications including ultra-sensitive optical detectors.

To this end, different types of optical antennas have been widely investigated to date [3-6]. However, in these nanoantennas, if using a simple architecture, the field enhancements are not at a maximum feasible level, or they have complicated 3D architectures and/or very sharp tips, which are technically challenging to fabricate. In addition, unfortunately, these specially shaped antennas have not been practically useful for infrared (IR) applications. Since the resonance wavelength is proportional to the antenna length, antennas with very long side lengths are required for the near infrared (NIR) and mid infrared (MIR) applications. Furthermore, these optical antennas exhibit either single resonance or multiple resonances with narrow bandwidths. Therefore, they are not suitable for multiband applications.

In this study, we designed and modeled optical antennas based on simple comb-shaped split ring architectures (Figure 1 inset) that enable resonance behavior at much longer wavelengths than the single dipoles or array of such dipoles with the same side lengths along the incident field polarization. In the proposed comb-shaped designs, the field intensity enhancements inside the gap regions are further found to be significantly enhanced compared to the cases of single and array of dipoles, despite operating on an electrical side length significantly reduced with respect to their resonance wavelengths [7]. Moreover, multiple spatial field localizations with multi-band resonance field enhancement spectrum are observed inside the gap regions.

We numerically studied and systematically simulated comb-shaped split-ring antennas made of gold on silica with a varying number of comb teeth using a finite-difference time-domain (FDTD) solver (Lumerical) and compared their performances with those of a single and array of dipoles. In our simulations, we illuminated antennas from the substrate (silica) side with a plane wave polarized along the long axis of a dipole or a comb tooth and examined the field enhancements at the gap center points over a wide wavelength range. In comb-shaped split-ring antenna simulations, the main resonance wavelength is found to red-shift as the number of comb teeth is increased. Also additional resonances are observed to emerge at shorter wavelengths as a result of adding comb teeth.

Figure 1 shows an exemplary design result here, with each of the comb teeth having $100 \mathrm{~nm}$ long, $40 \mathrm{~nm}$ thick and $40 \mathrm{~nm}$ wide arms, and each gap being $30 \mathrm{~nm}$ wide (thus with a side length of $230 \mathrm{~nm}$ ). In Figure 1, the field intensity enhancement spectrum of two-teeth comb-shaped split-ring antenna calculated at its gap center points is presented. Here multiple resonance peaks are observed and the maximum intensity enhancements are found to be 2163.7 and 1113.5 (compared to incident unitary intensity) at the outer and the inner gap center points, respectively, in a gap of about $\lambda / 106.7$ using an electrical length of about $\lambda / 13.9$ at $\lambda=3200 \mathrm{~nm}$ resonance wavelength. 

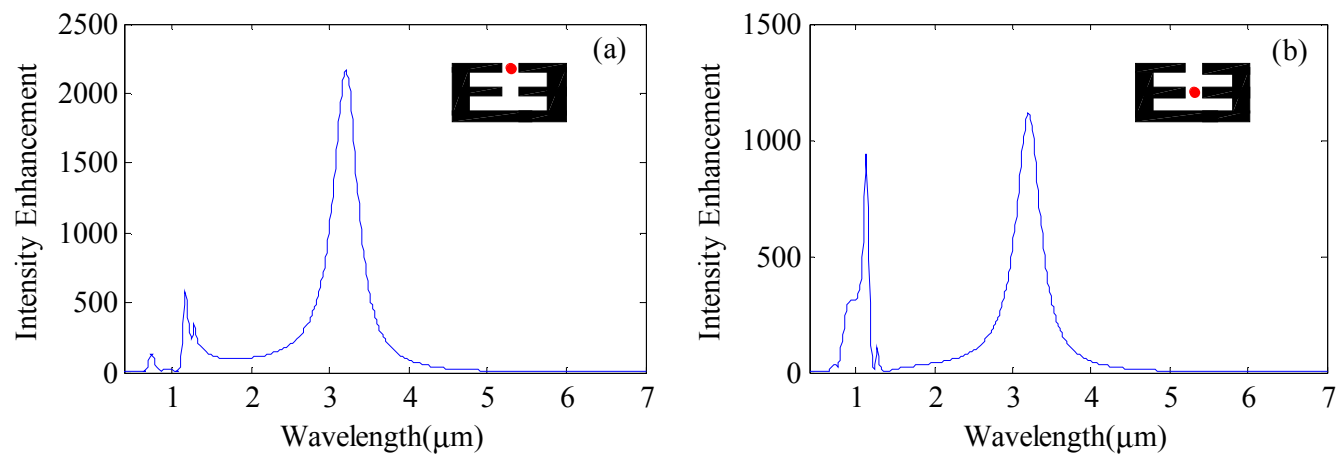

Fig. 1. Field intensity enhancement spectrum of two-teeth comb-shaped split-ring nanoantenna calculated at the center points of (a) the outer and (b) the inner gap covering NIR and MIR. Here, the outer and inner gap centers are marked with red dots in the corresponding inner drawings.

On the other hand, for a single dipole nanoantenna with the same arm length, thickness and width, a single resonance is observed and the peak field intensity enhancement is only 294.1 (in a gap of about $\lambda / 24.7$ using an electrical length of about $\lambda / 3.2$ ) at $\lambda=740 \mathrm{~nm}$ resonance wavelength. The field intensity enhancement spectrum of a single dipole nanoantenna is illustrated in Fig.2.

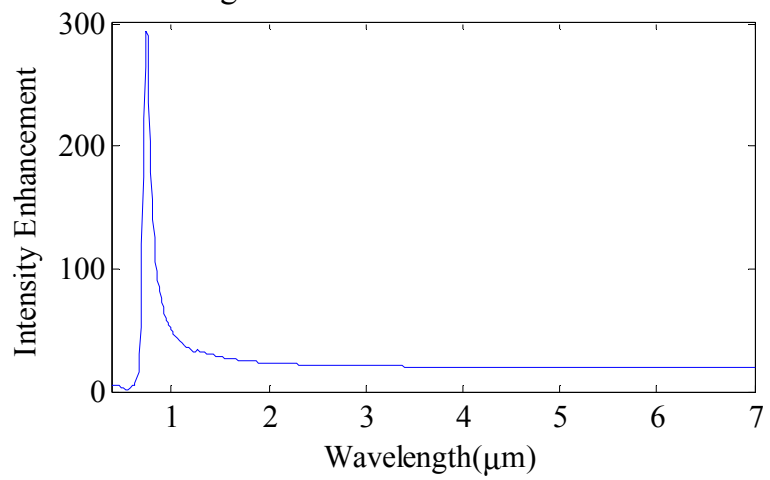

Fig. 2. Field intensity enhancement spectrum of a single dipole nanoantenna calculated at its gap center point.

In conclusion, in this work we present the resonance behavior of comb-shaped split-ring optical antennas. We show that total field intensity enhancement is larger in the comb architecture than those in the dipole cases using the same side lengths. The proposed comb antennas enable localization of NIR and MIR light within a region of tens of nanometers at an unprecedented intensity enhancement level. We believe that such comb-shaped split-ring nanoantennas are promising for applications that require intense optical spots beyond the diffraction limit in NIR and MIR and/or in those that require multi-band strong field localizations.

[1] D. K. Gramotnev and S. I. Bozhevolnyi, "Plasmonics beyond the diffraction limit," Nat. Photonics 4, 83-91 (2010).

[2] L. Novotny and N. van Hulst, “Antennas for light,” Nat. Photonics 5, 83-90 (2011).

[3] H. Fischer and O. J. F. Martin, "Engineering the optical response of plasmonic nanoantennas," Opt. Express 16, $9144-9154$ (2008).

[4] H. Tamaru, H. Kuwata, H. T. Miyazaki, and K. Miyano, "Resonant light scattering from individual Ag nanoparticles and particle pairs," Appl. Phys. Lett. 80, 1826-1828 (2002).

[5] R. M. Bakker, A. Boltasseva, Z. Liu, R. H. Pedersen, S. Gresillon, A. V. Kildishev, V. P. Drachev, and V. M. Shalaev, "Near-field excitation of nanoantenna resonance," Opt. Express 15, 13682-13688 (2007).

[6] J. Yang, J. Zhang, X. Wu, and Q. Gong, "Electric field enhancing properties of the V-shaped optical resonant antennas," Opt. Express 15, $16852-16859$ (2007).

[7] V. T. Kilic, V. B. Erturk, and H. V. Demir, "Optical antenna of comb-shaped split ring architecture for increased field localization in NIR and MIR,” Opt. Express 21, 29455-29461 (2013). 